\title{
Volumetric Solar Heating of Nanofluids for Direct Vapor Generation
}

\section{George $\mathrm{Ni}^{\mathrm{a}}$, Nenad Miljkovic ${ }^{\mathrm{a}, 1}$, Hadi Ghasemi ${ }^{\mathrm{a}, 2}$, XiaopengHuang ${ }^{\mathrm{a}}$, Svetlana V.} Boriskina $^{\mathrm{a}}$, Cheng-Te Lin ${ }^{\mathrm{a}, 3}$, JianJianWang ${ }^{\mathrm{a}}$, Yanfei Xu ${ }^{\mathrm{a}},{ }^{\mathrm{a}}$ M. MahfuzurRahman ${ }^{\mathrm{b}}$, TieJunZhang ${ }^{\mathrm{b}}$, and Gang $\mathrm{Chen}^{\mathrm{a}, *}$

\author{
Affiliation Addresses: \\ ${ }^{a}$ Department of Mechanical Engineering, Massachusetts Institute of Technology, 77 \\ Massachusetts Ave, Cambridge, Massachusetts 02139 \\ ${ }^{b}$ Department of Mechanical and Materials Engineering, Masdar Institute of Science and \\ Technology, Masdar City, Abu Dhabi, United Arab Emirates
}

\section{Present Addresses:}

${ }^{1}$ Department of Mechanical Science and Engineering, University of Illinois, Urbana, Illinois, 61801, USA

${ }^{2}$ Department of Mechanical Engineering, University of Houston, Houston, Texas, 77204, USA

${ }^{3}$ Key Laboratory of Marine New Materials and Related Technology, Zhejiang Key Laboratory of Marine Materials and Protection Technology, Ningbo Institute of Material Technology \& Engineering, Chinese Academy of Sciences, Ningbo, 315201, China

* Address post-published correspondence to: gchen2@mit.edu Address refereeing and publishing correspondence to: georgeni@mit.edu 


\section{Abstract}

2 Traditional solar-thermal receivers suffer from high surface temperatures, which increase

3 heat losses to the surroundings. To improve performance, volumetric receivers based on 4 nanoparticles suspended in liquid (nanofluids) have been studied as an approach to 5 reduce surface losses by localizing high temperatures to the interior of the receiver. Here, 6 we report measured vapor generation efficiencies of $69 \%$ at solar concentrations of 10 7 suns using graphitized carbon black, carbon black, and graphenesuspended in water, 8 representing a significant improvement in both transient and steady-state performance 9 over previously reported results. To elucidate the vapor generation mechanism and 10 validate our experimental results, we develop numerical and analytical heat transfer

16 Keywords:nanofluid, vapor generation, steam generation, solar energy, nanoparticle, volumetric receiver models that suggest that nanofluid heating and vapor generation occur due to classical global heating of the suspension fluid. This work demonstrates highnanofluid-assisted vapor generation efficiencies with potential applications in power generation, distillation, and sterilization.

\section{Introduction}

Traditional solar-thermal receivers consist of surface absorbers that convert the majority of the incoming solar radiation into heat while minimizing thermal re-radiation loss.[112] Although these receivers have high photothermal conversion efficiencies, surface absorbers are ill-suited for heating carrier fluids because the heat generation is separated from the fluid to be heated. At high solar concentrations (>50 suns), such as those used in industrial-scale solar thermal power plants,[13-21] a large temperature difference forms between the absorber and the fluid, leading to high surface temperatures and high radiative losses. One approach to minimizing the absorber-to-fluid temperature difference is to use volumetric absorption within the carrier fluid itself, which has been predicted to lead to a 5-10\% increase in photothermal efficiency.[17,22,23] Volumetric absorbers such as porous media,[24-29] gas-particle suspensions,[22,30-34] molten salts, $[1,6]$ and nanoparticles suspended in fluids (nanofluids) $[13,15-21,35,36]$ have been used to minimize surface temperatures of receivers, thereby reducing the receiver heat losses. Volumetric absorbers can have surface temperatures lower than even the bulk fluid temperature, [17,36,37] otherwise known as thermal trapping.[24,26,38]

Recently, nanofluids have been introduced as an attractive approach to direct steam generation using solar energy viananoparticleabsorption.[22,30,32,34,37,39]In addition, nanofluids have been shown to enhance critical heat flux in certain boiling applications.[40,41]Neumann et al. demonstrated the use of nanofluids for direct water vapor generation, $[6,42]$ reaching device efficiencies of $24 \%$ at solar concentrations of 1000 suns $\left(1\right.$ sun $\left.=1 \mathrm{kWm}^{-2}\right)$. Although a novel approach, the exact mechanism of vapor generation has been debated over the past few years.[6,35,36] Twopotential mechanisms have been proposed to explain the vapor generation results.[36,37,43] 
1 In one mechanism, nanoparticles isolate the heat generation to very near the particle-

2 liquid interface in a non-equilibrium manner such that the surrounding bulk fluid remains 3 cold while the particle heats up to a temperature which nucleates a vapor bubble locally. 4 Several researchers have studiednanoscale-to-microscale bubble formation surrounding 5 individual and arrayed nanoparticles. Lukianovaet al. conducted pulse-laser illumination 6 of gold nanoparticles to show that a critical laser fluence equivalent to $3 \times 10^{8}$ suns was 7 required before bubble formation initiated.[20,38,43-45] Fanget al, performed 8 continuous laser illumination experiments of gold nanoparticles on a substrate and 9 observed a similarly high nanobubble formation intensity threshold, on the order of $3 \times 10^{7}$ 10 suns.[37,46] Lombardet al. modeled theoretically the kinetics of nanobubble formation around gold nanoparticles, [36,42] showing that an intensity of $\sim 1 \times 10^{10}$ suns was required to nucleate a bubble. Though nanobubble formation has been observed, a combined optical absorption and heat conduction model using achievable illumination intensities does not give the required temperature differential.[6,47,48]

In the second mechanism, nanoparticles rapidly reach equilibrium with the surrounding fluid, and vapor generation is purely due to the rise in temperature of the bulk fluid.[2,36,43,49,50] Several experiments have shown that the interparticle fluid temperatures can reach as high as the spinodal decomposition temperature of water (594 $\pm 17 \mathrm{~K}$ ) before bubble formation.[20,43-45,51] In addition, Keblinski and Cahill simulated an array of 5000 nanoparticles, and found that two time scales exist in the heating profile of nanofluids.[46,52] They found that heating on the macroseconds scale is due to global heating of the fluid, but on the nanoscale the heating is confined near to the nanoparticle. Finally, a recent work by Hogan et al has focused on using high intensity lasers $\left(\sim 10^{6} \mathrm{~W} / \mathrm{m}^{2}\right)$ to show the effect of light scattering leading to localized absorption on the direct vapor generation from nanofluids. They simulated light propagation through the nanofluid, and compared experimentally with nanoparticles of varying scattering cross sections. In their work, they concluded that Fourier-law heat conduction adequately describes the nanoparticle-based direct steam generation.[2,36]

Based on the previous studies, there exists a need to 1) increase the efficiency of the direct solar vapor generation process to make the technology more competitive with existing solar vapor generation techniques, $[6,47,48] 2)$ seek solutions that utilize the full spectrum of solar energy at lower optical concentrations ( $\leq 10$ suns) than previous work to achieve commercial viability and minimize system cost,[2,49,50,53] and 3) gain a better understanding of the physical mechanisms governing solar vapor generation. Through rational design and detailed experiments, we show highly efficient direct vapor generation $\left(69 \pm 4 \%\right.$ at $\left.80^{\circ} \mathrm{C}\right)$.We attain these results using water based nanofluidsolar receivers at low optical concentrations ( $\leq 10$ suns), in comparison to all previous work, which used high intensity lasers or high optical concentration solar flux. A solar concentration of 10 suns is approximately the highest achievable without active suntracking, via non-imaging optics.[51,54]Insome concentrated solar plants (CSP) the optical collection field can comprise up to $30 \%$ of the total installed cost.[52,55] In addition, we report that well-dispersed nanofluids can lead to higher vapor generation in transient conditions. Furthermore, through a consistent set of numerical simulation, analytical modeling, and experimental validation, we clarify that the solar vapor 
1 generation of nanofluids is in fact due to global heating of the bulk fluid and related 2 classical evaporation phenomena. This work advances a direct solar vapor generation 3 platform that promises to be low cost and has potential for a wide-range of solar-based

4 applications such as power generation,[2,56] distillation,[6,32] and sterilization.[53,57]

\section{Methods and Materials}

6 To study the effect of different nanofluids on the receiver efficiency, we performed solar 7 vapor generation experiments on a custom-built lab-scale receiver. To supply solar 8 energy to the nanofluid samples, a solar simulator was used in conjunction with a Fresnel 9 lens and aperture to generate and focus concentrated solar light (Fig.1a). The nanofluid 10 container was constructed out of two concentric acrylic tubes, with a layer of aerogel particles in between to serve as an insulator to minimize radial heat losses (Fig.1b). The aerogel particles were sealed from the environment with acrylic discs. The nanofluid was exposed to the ambient to vent the vapor. No insulation was used over the top of the nanofluid, which maximized the evaporation efficiency by allowing faster vapor diffusion. Four E-type thermocouples were inserted into the nanofluid container to measure the fluid temperature at different distances from the nanofluid-air interface. As the nanofluid evaporated, the fluid level dropped below each thermocouple, allowing temperature measurement of the liquid-vapor interface location. The mass loss was measured using a high accuracy weight scale (see Supporting Information, section S1).

20 Carbon based nanoparticles are significantly lower cost than metal nanoparticle suspension, and have better broadband solar absorptance.[58]Three different highly absorbing nanofluids were synthesized for this work: graphitized carbon black (GCB, Fig.1c), carbon black (CB, Fig.1d), and graphene particles suspended in water (Fig. 1e). The nanofluids were created by sonicating $0.5 \mathrm{wt} \%$ of the various nanoparticles in distilled water for 1 hour. We chose the nanoparticle concentration to be $0.5 \mathrm{wt} \%$ based on previous works in studying the effect of nanoparticle fraction on photothermal properties of nanofluids.[58]The GCB (Sigma-Aldrich, 699632-25G <500nm) and CB

28 (Cabot, Vulcan 9 N110) were commercially purchased. The graphenenanosheets were made using an electrochemically stimulated exfoliation process.[6,54] All three nanofluids appeared stably suspended throughout the duration of the test. During storage, the GCB nanofluid was stable for months at a time, whereas the graphenenanofluid was stable for over a year. The CB nanofluid was stable for periods less than a week.

33 To study the non-constant nature of solar irradiance during the day, both steady-state and 34 transient receiver efficiencies were measured. 

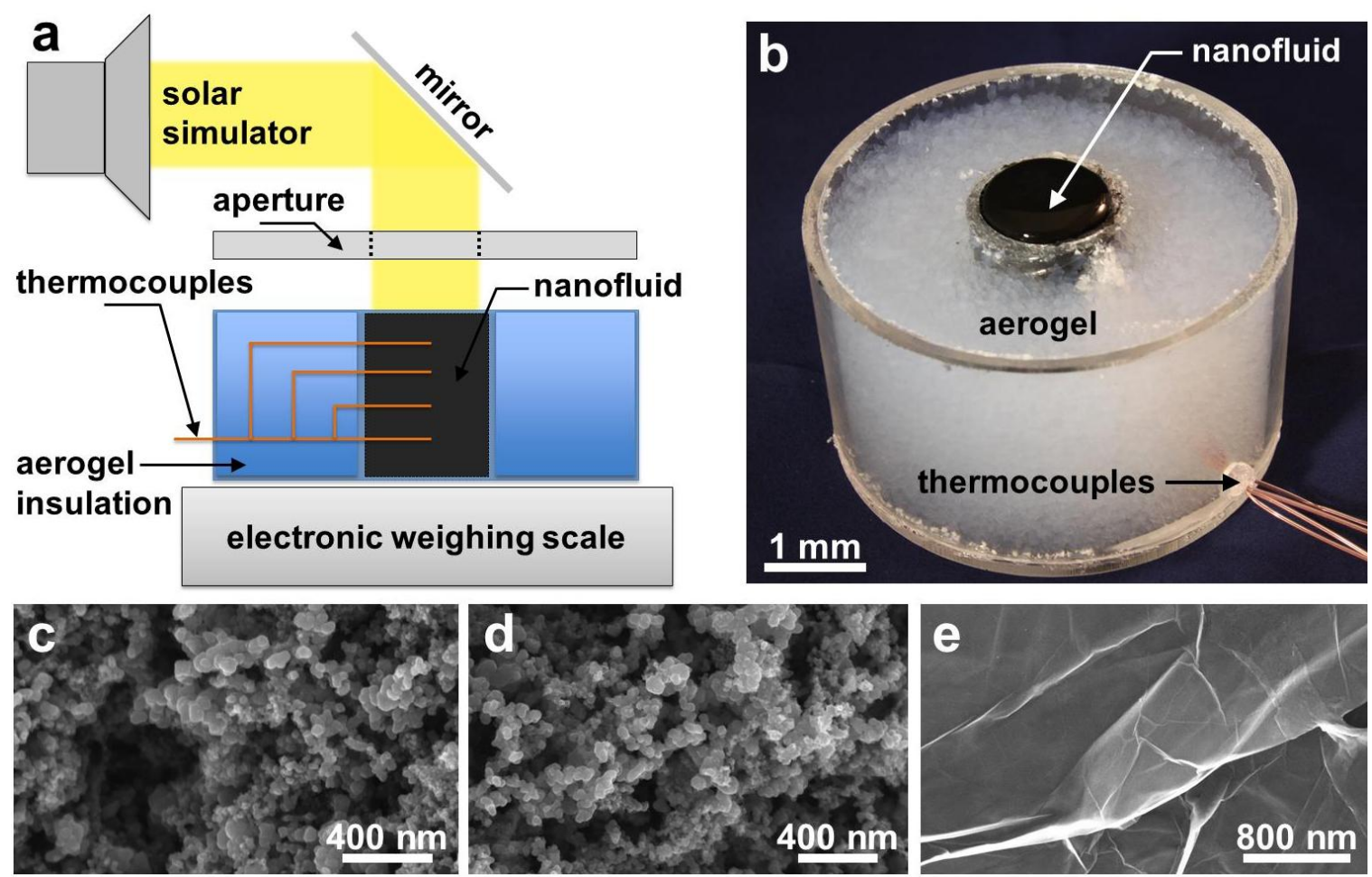

Figure 1. (a) Schematic of solar vapor generation device. (b) Image of the nanofluid container showing the aerogel insulation, black nanofluid, and thermocouple feed through. Scanning electron micrographs (SEM) of (c) graphitized carbon black, (d) carbon black, and (e) graphenenanoparticles. To obtain SEM images, the nanofluids were dehydrated prior to imaging.

The small cuvette measurements (Lumped Capacitance Model, Fig.6) consisted of a rectangular transparent cuvette (Plastibrand, PMMA) with dimensions $12.5 \mathrm{~mm} \times 12.5 \mathrm{~mm}$ $\mathrm{x} 45 \mathrm{~mm}(L \times W \times H)$. The cuvette was filled with GCB nanofluid, illuminated from the side with the solar simulator. Three E-type thermocouples (Omega Engineering, TT-E40-SLE-50) inserted through the cuvette walls measured the nanofluid temperature at different heights in the cuvette. One additional thermocouple is placed above the liquidvapor interface, and is shielded from direct illumination with aluminum foil. Various solar concentrations were used $\left(1-10 \mathrm{kWm}^{-2}\right)$, and the temperatures were recorded until steady state was reached. For additional information about the small cuvette experiment, see Supporting Information, section S2.

\section{3. Results}

18 Figure 2 shows the experimental mass change of the nanofluid container as a function of time for each of the studied nanofluids at 10 sun illumination. The absolute mass change rate $(|d m / d t|)$ started at zero and gradually increased with time due to the photothermal conversion of solar radiation to enthalpy of evaporation. As the nanofluid absorbed more solar radiation, the temperature of the bulk nanofluid solution gradually increased in conjunction with the evaporation rate. After an initial heating period of $\approx 4000$ seconds, the system reached steady-state operating conditions where the evaporative and parasitic heat losses balanced the absorbed solar radiation. The evaporation efficiency at steady- 
1 state conditions was determined by dividing the gained enthalpy in the generated vapor

2 by the total incoming solar radiation input,

$$
\eta=\frac{\dot{m} h_{f g}}{Q_{s} A},
$$

4 where $\dot{m}$ is the steady-state vapor mass flux, $h_{f g}$ is the latent heat of vaporization for 5 water at $1 \mathrm{~atm}\left(2.257 \mathrm{MJkg}^{-1}\right), A$ is the area of the aperture $\left(4.95 \mathrm{~cm}^{2}\right)$, and $Q_{S}$ is the total 6 incoming solar flux $\left(10 \mathrm{kWm}^{-2}\right)$ after concentrating optics, hence the efficiency reported 7 is an internal efficiency. The steady-state efficiency was determined by using the data 8 where the mass loss is linear to within an $\mathrm{R}^{2}$-value of 0.999 . Although the addition of 9 nanoparticles to water has been reported to change the thermophysical properties such as 10 heat capacity[51,55] and thermal conductivity,[56,59,60] the small concentration of 11 nanoparticles used in this study $(0.5 \mathrm{wt} \%, 0.23 \mathrm{vol} \%)$ was determined to have a 12 negligible effect on both properties.[32,61]

13 The system evaporates nanofluids similarly to a continuous process. The addition of 14 replenishing fluid would add two details to our analysis: 1) conduction of heat to the 15 underlying and flowing liquid, and 2) use of some of the absorbed solar energy to heat 16 the nanofluid up to operating temperature (sensible heating). The receiver had already 17 reached within $2 \%$ of steady-state evaporation, while thermocouples showed the 18 underlying liquid to be near room temperature $\left(<30^{\circ} \mathrm{C}\right)$. This shows that conduction into 19 the liquid is not a dominant heat loss mechanism. Thesensible heat increase in the 20 generated vapor phase $\left(\dot{m} c_{p} \Delta T\right)$ was small ( 7\%)compared to the latent heat of 21 vaporization $\left(\dot{m} h_{f g}\right)$, and was purposely excluded to conservatively estimate the 22 efficiency. 


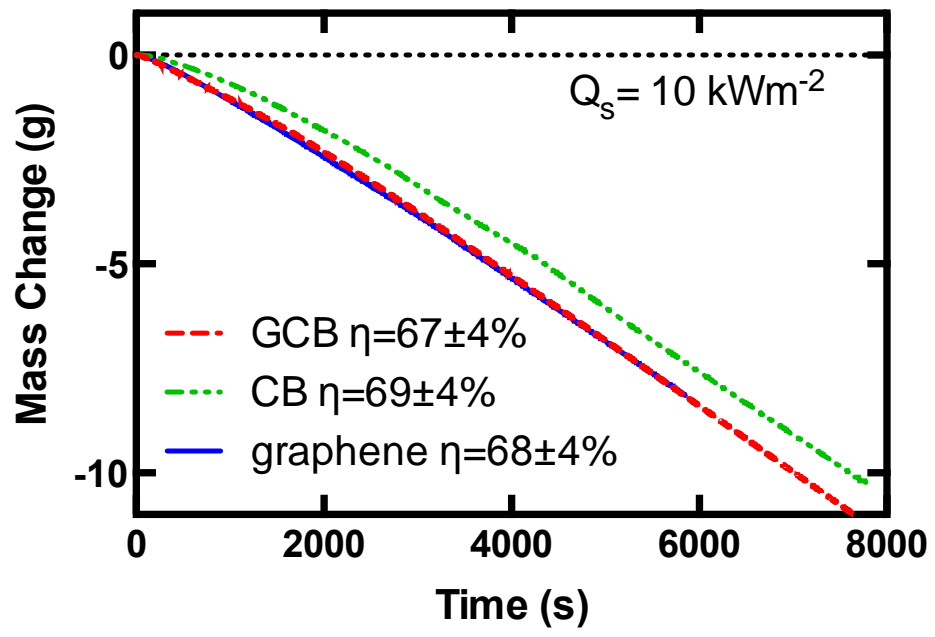

2 Figure 2.Nanofluid receiver mass change as a function of time for the carbon black (CB), 3 graphitized carbon black (GCB), and graphenenanofluids while illuminated by 10 suns of 4 radiation $\left(Q_{s}=10 \mathrm{kWm}^{-2}\right)$. At steady-state $(t>4000 \mathrm{~s})$ the mass change rate was 5 approximately equal for all of the nanofluids $\left(d m / d t=\dot{m} \approx-1.5 \times 10^{-3} \mathrm{gs}^{-1}\right)$.

The steady-state evaporation efficiency was approximately the same for all three nanofluids tested $(\eta \approx 69 \%)$, with all calculated values being within the measurement uncertainty $( \pm 4 \%)$. Of the losses from our system, radiation was calculated to be $4 \%$. The measured specular reflectivity of the nanofluids was <1\% (see Supporting Information, section S5). The losses into the container were modeled using a COMSOL simulation and matching the boundary conditions to the embedded thermocouples. The conduction into the bulk underlyingnanofluid was $\approx 9 \%$, and thelosses to the surrounding aerogel insulationand ambient were $\approx 12 \%$. The air convection from the evaporating nanofluid surface accounted for $\approx 3 \%$ of the total losses (see Supporting Information, section S3). Transmission losses were not present for the nanofluids studied here due to all of the incoming radiation being absorbed prior to reaching the bottom of the nanofluid container.

It is important to note that the $69 \%$ efficiency includes the incident power on the receiver, but not the losses from the optics. If a Fresnel lens with modest optical efficiency of $\approx 83 \%$ were used to supply concentrated solar light,[57,62] a system vapor generation efficiency of $57 \%$ would be achieved. Even with the additional optics loss included, our reported vapor generation efficiency of $57 \%$ is higher than achieved in a previous study $(\approx 24 \%)$.[6,57]In addition, our nanofluids-based receiver utilized a much lower solar concentration (10x vs. 1000x), one achievable with less stringent tracking requirements and lower cost components.[36,51]

Since the measured steady-state efficiencies for each nanofluid were approximately identical, it can be inferred that the global absorptanceof the different nanofluids are similar. Furthermore, the high steady-state vapor conversion efficiency shown here can be attributed to the high absorptivity of the nanofluid in the solar spectrum, and the 
unrestricted vapor extraction. In addition, the utilization of low thermal conductivity aerogel insulation $\left(\sim 0.02 \mathrm{Wm}^{-1} \mathrm{~K}^{-1}\right)$ helped to minimize side loses and allow for a 3 majority of the photothermal energy conversion to be utilized for water phase change. In 4 a larger application-scale nanofluids-based solar receiver, the side losses would be 5 smaller than in the lab-scale device due to a lower surface-to-volume ratio, and even 6 higher efficiencies are potentially achievable.

To examine the transient performance of the nanofluid receiver, we compared the mass change on a smaller time scale $(0<t<300 \mathrm{~s})$. Each of the nanofluids was first measured under dark conditions for 10 minutes, to ensure that the nanofluid temperature was consistent with the lab ambient temperature. The cover was removed from the aperture, and data acquisition was initiated. Figure 3(a) shows the mass loss as a function of time during the transient period for the threenanofluids. The GCB-based nanofluid evaporated the most water during the transient period $(\approx 0.3 \pm 0.001 \mathrm{~g})$, followed by the graphene $(\approx 0.22 \pm 0.001 \mathrm{~g})$ and regular CB nanofluids $(\approx 0.1 \pm 0.001 \mathrm{~g})$. The transient performance of the nanofluidswas related to how well-dispersed the nanoparticles in the fluids were. The CB nanofluid was noticeably less well-dispersed, and the meniscus was more transparent with particle agglomerates discernible by eye. Nanofluids with welldispersed particles generated heat closer to the liquid-vapor interface, and had a higher interfacial temperature and overall evaporation rate. The nanofluiddispersity is shown later to be related to the extinction coefficient of the nanofluid. The reason for the variable nanofluid dispersions is due to the different zeta potentials of the nanoparticles in the water solution, which is related to nanofluid stability. Nanofluids with high magnitude of zeta potential (negative or positive) are electrostatically stabilized, while nanofluids with low magnitude zeta potentials tend to agglomerate. $[59,60,63]$ CBnanofluids have been reported to have a zeta potential of only $\approx-10 \mathrm{mV},[61,63,64]$ resulting in agglomeration and lack of stability, while the graphene and GCB nanofluids reportedly have lower zeta potentials (higher magnitude, $\approx-40 \mathrm{mV}$ ), [36,62] resulting in better nanofluid stability, less agglomeration, and enhanced transient performance.
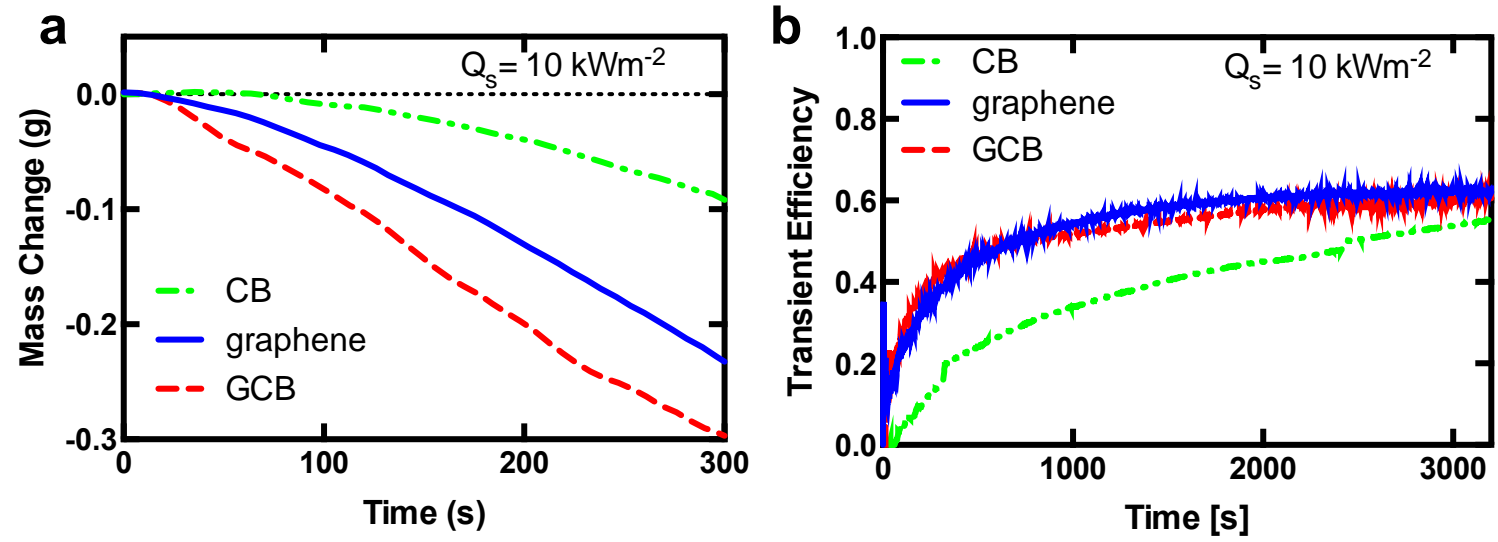

Figure 3. (a) Mass change in transient conditions and (b) transient efficiency as a function of time for the $\mathrm{CB}, \mathrm{GCB}$, and graphenenanofluids while illuminated by 10 suns of solar radiation $\left(Q_{s}=10 \mathrm{kWm}^{-2}\right)$. The GCB-based nanofluid had the highest transient evaporation efficiency, followed by the graphene and regular CB nanofluids. 
1 To quantify the transient performance in terms of vapor generation efficiency, we define

2 a transient efficiency, $\eta_{T}$, as the total amount of water evaporated since illumination

3 began divided by the total solar energy incident on the nanofluid receiver during that time

4 interval,

$$
\eta=\frac{\int_{0}^{t} \dot{m}\left(h_{f g}+c_{p, l} \Delta T\right) d t}{\int_{0}^{t} Q_{s} A d t}
$$

6 where $c_{p, l}$ is the specific heat of liquid water $\left(4.19 \mathrm{kJkg}^{-1} \mathrm{~K}^{-1}\right)$, and $\Delta T$ is the temperature 7 rise of the liquid prior to evaporation. The transient efficiency depends on the temporal 8 length of the measurement, but is appropriate when considering varying solar power over 9 the course of the day. Despite the GCB nanofluid reaching steady-state faster (Fig.3a), 10 the graphenenanofluid reaches a similar transient efficiency $(69 \pm 4 \%$ for $0<t<6000 \mathrm{~s})$. 11 The transient efficiencies of GCB and graphene nanofluids are 7\% higher than that of $\mathrm{CB}$ 12 nanofluid. At shorter measurement times, this transient efficiency discrepancy increases 13 as shown in Fig. $3 b$. The measured transient receiver efficiency of $69 \pm 4 \%$ can again be 14 coupled with a modest Fresnel lens optical efficiency of $83 \%,[57,65]$ giving a system 15 transient vapor generation efficiency of 57\%.

16 It is important to note that the identical steady-state performance does not indicate that all 17 three nanofluids will achieve the same performance in a given solar application. The 18 transient performance becomes crucial when choosing nanofluids for applications that 19 may have intermittent interruptions in illumination such as rolling cloud cover. Another 20 situation where transient performance becomes important is cases where solar tracking is 21 not used, such as in residential homes. In these cases the solar illumination angle is 22 constantly changing, and therefore the incoming radiation is changing throughout the 23 course of the day. In the case of transient incoming solar radiation, the GCB nanofluid 24 would perform the best due to its ability to reach steady state the fastest and generate the 25 most vapor in the transient period of operation.

\section{Transient Efficiency Model}

To explain the transient absorption mechanism, we developed an analytical heat transfer model to show that the transient efficiency is dependent on the extinction coefficient of the nanofluid, which is determined by the absorption and scattering characteristics of nanoparticles and their agglomerate size $\left(r_{o}\right)$. A previous study of nanofluids based on metal nanoparticles has shown a positive correlation between the nanoparticle extinction coefficient and nanofluid evaporation rates.[36,65] Since all the nanoparticles are carbon based, we mainly consider the effect of the agglomerate sizes, which were experimentally measured using optical characterization methods, and the dependence of calculated

35 evaporation flux on the extinction coefficient was determined using the developed 36 analytical model.

37 The nanoparticle agglomerate sizes were determined using dynamic light scattering 38 (DLS) and optical microscopy, depending on the agglomerate size. The GCB 
nanoparticles were well dispersed, and had smaller agglomerates $\left(r_{o} \sim 110 \mathrm{~nm}\right)$ suitable for

2 DLS measurement. The CB nanoparticles are less well dispersed, and their agglomerates 3 were larger $\left(r_{o} \sim 5 \mu \mathrm{m}\right)$ and observable with optical microscopy (see Supporting 4 Information, section S6). The agglomerate sizes determined the particle density in the nanofluids, since the volume fractions were the same.

Once the nanoparticle agglomerate sizes were determined, an extinction coefficient was 7 calculated for GCB and CB nanofluids, using Mie theory in the independent scattering 8 regime,[63,66] and indices of refraction from literature (see Supporting Information, section S6).[63,64,67] The calculated scattering and absorption cross sections of nanoparticle agglomerates were of similar magnitudes. To approximate the heat generation, the total extinction coefficient calculated was used in the heat generation term for Eq.3 in our transient efficiency model shown later. In the event that forward scattering dominates back scattering, as is the case with the studied nanoparticle agglomerates, our calculations will under predict the difference in transient efficiency. Figure 4. shows the calculated efficiency factors $Q_{\text {ext }}, Q_{a b s}, Q_{s c a}$ for extinction, absorption, and scattering. Using the efficiency factors, the extinction coefficients for GCB and $\mathrm{CB}$ were calculated and found to differ more than two orders of magnitude. The estimated extinction coefficients for GCB and $\mathrm{CB}$ nanofluids were $5.6 \times 10^{5} \mathrm{~m}^{-1}$ and $1.6 \times 10^{3} \mathrm{~m}^{-1}$ respectively. Again, the difference in extinction coefficients is largely due to the particle (agglomerate) number density, which given a same amount of material is related to the stability of the suspension.

a

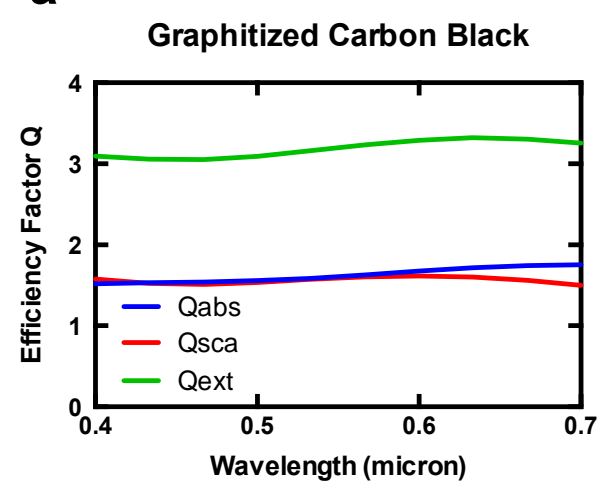

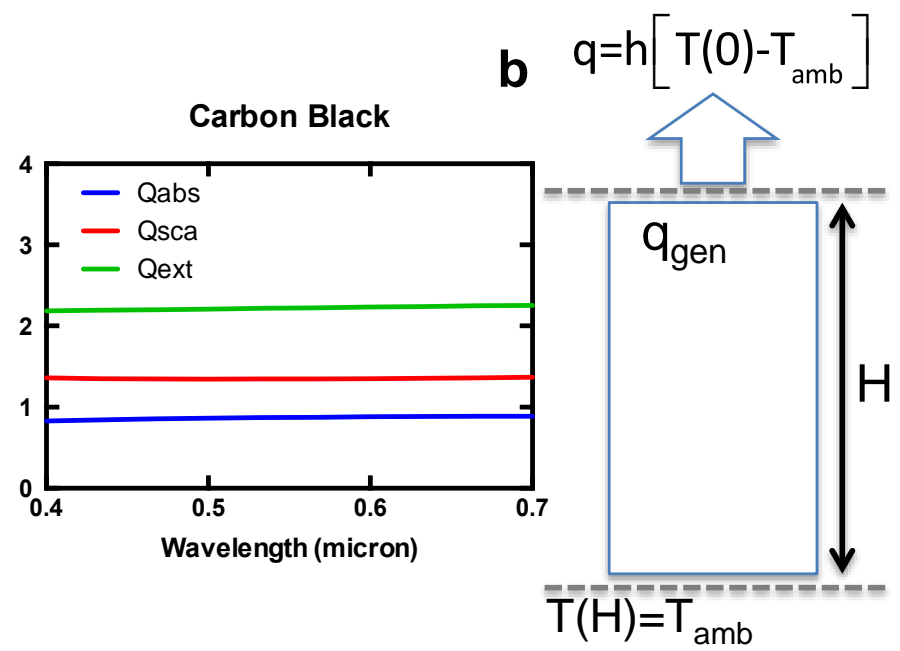

3 Figure 4. (a) Efficiency factors for the nanoparticles calculated using Mie theory. $Q_{\text {ext }}, Q_{a b s}, Q_{s c a}$ are the efficiency factors for extinction, absorption, and scattering. The carbon black has a smaller extinction efficiency than the graphitized carbon black. (b) A visual schematic of the transient efficiency model.

Using the estimated extinction coefficients of the nanofluids, we constructed an analytical heat transfer model of the nanofluid receiver to determine the effect of extinction 
1 coefficient on the transient performance. Our model neglects convection and considers 2 transient conduction effects coupled with absorption. Only conduction is considered 3 because the heat generation occurs at the top end of the receiver, thereby minimizing 4 natural convection inside. The weaker extinction coefficient shifts the heat generation 5 deeper into the nanofluid receiver, and reduces the temperature of the nanofluid-air 6 interface. This ultimately reduces the evaporation efficiency of the device. A schematic 7 of the transient efficiency heat transfer model can be seen in Fig. 4c. The analytical 8 model is shown in Eq. 3, where $\lambda$ is an exponential constantfor heat generation, and $q_{0}$ is 9 the incident light intensity.

$$
\begin{gathered}
\qquad \frac{\partial \Theta(x, t)}{\partial t}=\alpha_{x} \frac{\partial^{2} \Theta(x, t)}{\partial x^{2}}+\frac{q_{0} \lambda e^{-\lambda x}}{\rho c_{\mathrm{p}}} \\
\text { Boundary Condition \#1: } \Theta(H, t)=0 \\
\text { Boundary Condition \#2: }-k_{x} \frac{\partial \Theta(0, t)}{\partial x}+h \Theta(0, t)=0 \\
\text { Initial Condition: } \Theta(x, 0)=0
\end{gathered}
$$

10 The boundary and initial conditions for the heat transfer model are: 1) convectively 11 cooled temperature bath on one end (the evaporation side, $x=0$ ), with $h\left(150 \frac{\mathrm{W}}{\mathrm{m}^{2} \mathrm{~K}}\right)$ fitted 12 from the COMSOL simulation of the nanofluids receiver, and 2) constant ambient 13 temperature at the other side $(x=H)$, and 3) initially, the nanofluid receiver is at ambient 14 temperature. The model is solved using the Green's function method. The results of the 15 simulation are shown below in Fig.5.
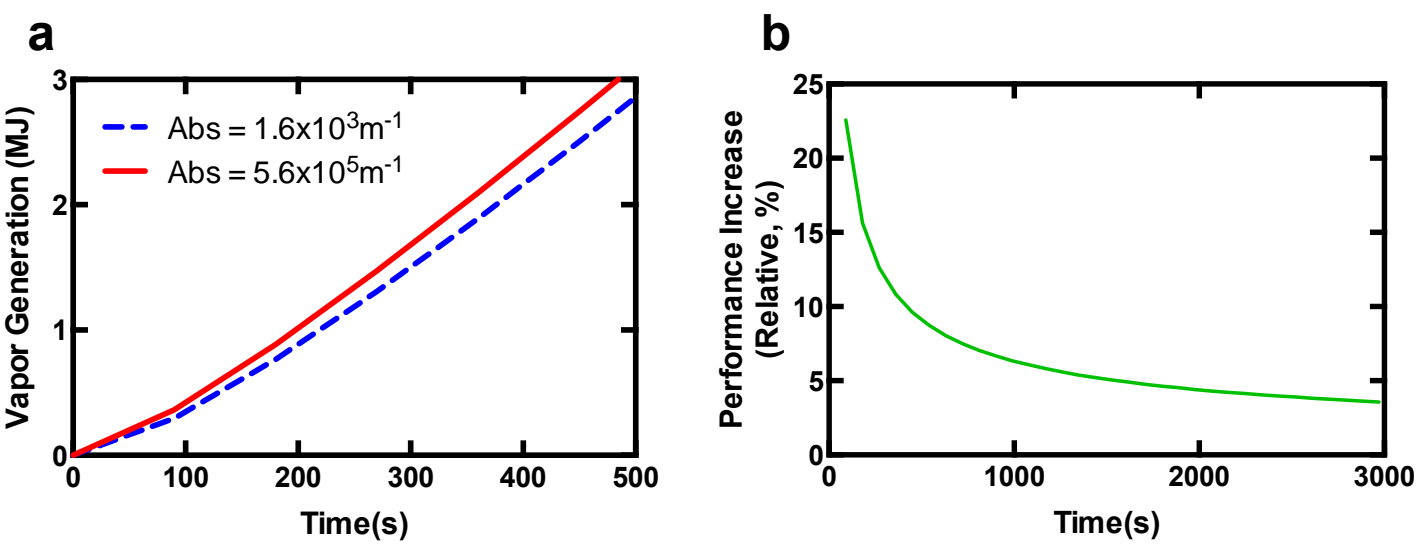

17 Figure 5. (a) The calculated energy loss through evaporation plotted as a function of time, 18 for nanofluids with different extinction coefficients. The higher extinction coefficient 19 leads to higher evaporation flux due to heat localization at the nanofluid-air interface and 
1 higher surface temperature. (b) The relative vapor generation increase in total evaporated

2 energy between the nanofluids considered in (a).

3 Figure 5a shows the calculated total water vapor generated from the nanofluid as a 4 function of time for the GCB and CB nanofluids. It can be seen that the nanofluid with 5 the larger extinction coefficient (GCB) has the higher vapor generation rate. To help 6 quantify the performance difference between the two nanofluids, Fig.5b shows the 7 relative vapor generation increase between the two nanofluids, which is defined below.

$$
\eta_{\text {relative }}=\frac{h \Theta_{G C B}(0, t)}{h \Theta_{C B}(0, t)}
$$

where $\eta_{\text {relative }}$ is the relative performance increase of the GCB nanofluid over the CB nanofluid. The performance increase is particularly significant shortly after illumination, and decreases over time. At 3000 seconds, the total vapor generated for the two nanofluids differs by about $4 \%$. This is smaller than the experimentally measured relative difference of $10 \%$ in the transient receiver efficiency (Fig.3). We attribute this discrepancy to the use of a constant heat transfer coefficient in the model, whereas in reality the evaporation rate will increase non-linearly with temperature. This is due to the non-linear dependence of vapor pressure, the driving force for evaporation, on temperature. Nonetheless, the experimental and model results show good agreement. This analytical model shows how heat localization due to a larger effective extinction coefficient in the nanofluid can increase the transient evaporation, corroborating recent work on metal particles $[36,68]$, but does not clarify the mechanism for vapor generation.

\section{Horizontal Illumination: Lumped Capacitance Model}

To provide insight into the experimental results, and support the mechanism of global fluid heating for vapor generation in these nanofluids, we conducted additional experiments and developed the corresponding model (see Supporting Information section S2). We show from the experiments and model that the evaporation heat transfer coefficients developed to model the evaporation of pure water can also be used to describe the evaporation behavior of nanofluids. We illuminated the nanofluids from the side to achieve uniform temperatures throughout the nanofluid. This allows us to utilize the lumped capacitance approximation in the model. The following assumptions were made: 1$)$ the fluid is isothermal throughout the cuvette $\left(B i=\bar{h} L / k_{w} \approx 0.1\right.$, where $B i, \bar{h}$, $L$, and $k_{w}$ are the Biot number, external heat transfer coefficient to air $\left(\bar{h} \approx 5 \mathrm{Wm}^{-2} \mathrm{~K}^{-1}\right)$, thickness length scale of the cuvette $(L \sim 10 \mathrm{~mm})$, and nanofluid thermal conductivity $\left(k_{w} \approx 0.6 \mathrm{Wm}^{-1} \mathrm{~K}^{-1}\right)$, respectively. 2 ) The boundary condition at bottom of the cuvette 33 was considered insulated. 3) The side walls are modeled as heated vertical plates undergoing natural convection to the surrounding ambient air, $[65,69,70]$ and radiative losses. 4) The top evaporating surface undergoes both natural convection and evaporation.[65] 5) All incident solar radiation is absorbed by the nanofluid $(\varepsilon \approx 1)$. The high solar absorption was validated by measuring the transmission of solar light 
1 through a nanofluid filled cuvette using a UV-VIS spectrophotometer (see Supporting 2 Information, section S5).

3 It is important to note that although our transient efficiency model showed that the fluid 4 temperature is in fact non-uniform and dependent on the extinction coefficient, the assumption of lumped capacitance in this model is still valid, due to the different illumination conditions. Furthermore, this model is not meant to further elucidate or resolve the transient performance discrepancy from sample to sample, but rather to give a 8 physical picture of the vapor generation process in terms of a global energy balance and validate the classical heat loss mechanisms present in the experiment.

10 Accounting for all of the heat transfer pathways, the differential equation for the bulk 11 nanofluid temperature, $T$ is (for full derivation, please see Supporting Information, 12 Section S2)

$$
\begin{aligned}
\rho_{w} c_{p} V \frac{d T}{d t}+\rho_{w} c_{p} T & \frac{d V}{d t} \\
& =q-\left(\overline{h_{s}} A_{s}+\bar{h}_{t} A_{t}\right)\left(T-T_{\infty}\right)-\varepsilon \sigma A_{T}\left(T^{4}-T_{\infty}{ }^{4}\right)-\overline{h_{e}} A_{t}\left(P(T)-\varphi P\left(T_{\infty}\right)\right),
\end{aligned}
$$

13

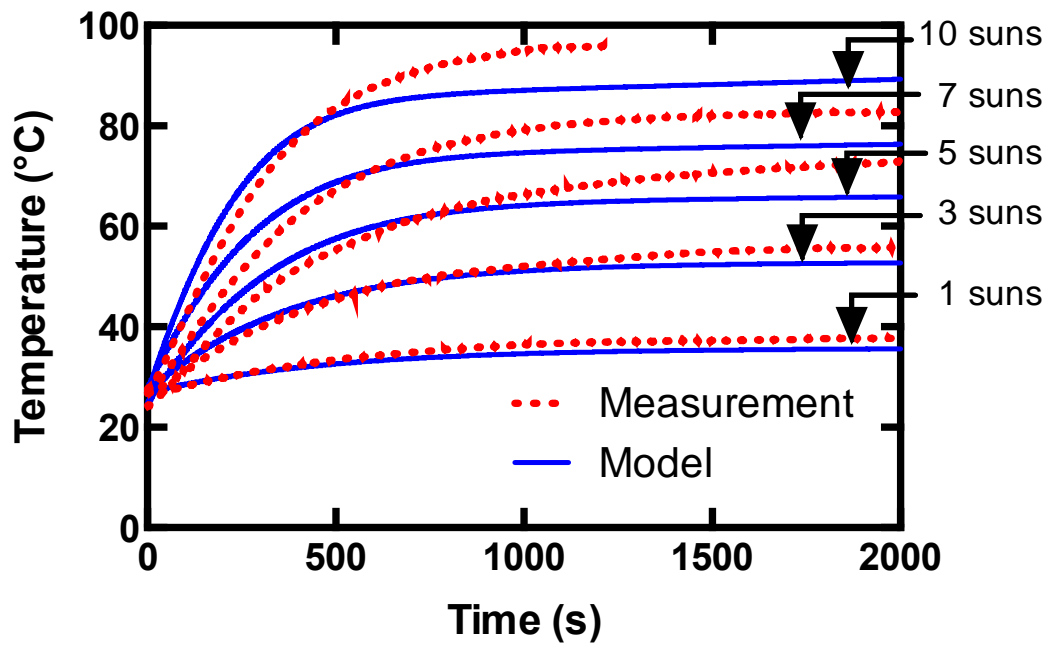

23 Figure6. Graphitized carbon black nanofluid temperature as a function of time for 5 24 different solar concentrations. The experimental results are shown in red solid lines, 25 while the analytical model results (Eq. 5) are shown in blue dotted lines. The bulk fluid 26 temperature was calculated by calculating the arithmetic mean of the four thermocouple 
1 probes in the nanofluid. The experimental error in the thermocouple measurement is

2 approximately $\pm 0.5^{\circ} \mathrm{C}$.

3 Figure 6 shows the experimental and model results of the nanofluid temperature as a 4 function of time for a range of solar concentration ratios ( $1<C<10$ suns, see Methods). 5 As the incoming solar light was absorbed by the nanofluid, the bulk fluid temperature 6 began to rise due to sensible heating of the nanofluid. As the nanofluid temperature 7 continued to increase, the evaporation rate and parasitic heat losses (i.e. natural 8 convection and radiation) began to dominate the energy transfer mechanisms, until the 9 steady state was reached ( $t>2000 \mathrm{~s}$ ) where all of the incoming solar energy was being converted to evaporation and parasitic heat losses.

The heat transfer model agrees well with the experimentally measured time-dependent temperature profile of the bulk nanofluid. This indicates that the fluid is directly heated via conduction by the absorbing nanoparticles at the surface and that vapor is not being generated at the nanoparticles themselves in a non-equilibrium fashion as described previously. If the vapor was indeed generated at the nanoparticle, and not the liquid-air interface, the bulk temperature profile would be reduced, due to the localized heat generation.

18 Our model becomes less accurate in steady-state operation $(t>2000 \mathrm{~s})$ due to the changing concentration of nanoparticles as water leaves the system, especially near the liquid-air interface. The evaporation of water left a concentrated layer of hydrophobic GCB particles, which formed a skin at the interface, and restricted evaporation. This reduced the evaporation heat transfer coefficient below that of pure water (used in the model), and contributed to the increasing temperature of the bulk fluid.[67] Furthermore, the latent heat of vaporization of nanofluids has been shown to potentially be significantly higher than the aqueous constituent alone,[68] leading to more energy required to evaporate the liquid water, lower evaporation rates, and higher steady-state temperatures. Although the lumped capacitance nanofluidmodel developed here does well at predicting the experimental behavior during nanofluidvapor generation, it fails to give a mechanistic understanding of the energy conversion mechanisms at the nanoparticle scale, which must be reconciled with additional modeling in order to gain a better understanding of the heat generation physics.

\section{6. Particle Heating Model}

33 To study the nanoparticle-fluid temperature difference, we used a 3D numerical 34 simulation (COMSOL) to model an array of nanoparticles distributed evenly in a fluid 35 medium. For such periodic structures, we can focus on the heat transfer in one unit cell to understand the entire structure. The COMSOL model consists of a particle-in-a-box, a single heated nanoparticle in a fluid domain(Fig.7a). The following details were used to construct the model. The dimensions of the box were based on the average nanoparticle spacing in the nanofluid, which for a $0.5 \mathrm{wt} \%$ GCB nanofluid was calculated to be $\approx 3 \mu \mathrm{m}$ for a nanoparticle radius of $250 \mathrm{~nm}$. The boundaries of the fluid box were insulated, due to symmetry (Fig.7b). A boundary heat flux was placed at the nanoparticle surface, which simulated the absorption of solar energy. The nanoparticle was assumed to be 
1 spherical, isothermal, and surrounded by liquid water. Only transient heat conduction 2 was considered at these small length scales. Non-equilibrium nanoscale heat conduction 3 effects were not considered due to the relatively high interfacial conductance at carbon4 water interfaces.[69,70]

5 At time scales on the order of a few seconds, the temperature variation across the fluid 6 box was negligible, $<0.01 \mathrm{~K}$. This is not surprising, as the spacing between nanoparticles 7 in the fluid is very small $(<3 \mu \mathrm{m})$, and the corresponding Fourier number is high $8 \quad\left(F o>10^{4}\right)$. This further supports a global temperature rise in the fluid medium as the 9 proposed mechanism of evaporation.[46] Only at very short time scales $(\sim \mu \mathrm{s})$ and high 10 solar intensities $\left(\sim 10^{5}\right.$ suns) can a temperature difference of $100 \mathrm{~K}$ be found over the fluid 11 box. This high solar concentration is roughly in agreement with the laser intensities 12 required for nanobubble formation in previous studies, and is larger than achievable solar 13 concentrations.[37,38,42]

14 To study the effect of overlapping thermal boundary layers of nearby nanoparticles on the 15 bulk fluid temperature, the particle separation distance $\left(2 L_{\text {fluid }}\right)$ was varied. In previous 16 works, models of a single nanoparticle in an infinite medium have been considered.[6,42] 17 However, this ignores the heating effects of nearby nanoparticles in a real fluid and is 18 only valid for short time scales where the individual heating profile has not reached the 19 neighboring particles.[46,71] Fig.7c shows the fluid temperature profile as a function of 20 normalized distance from the nanoparticle wall in the $\mathrm{x}$-direction for different particle 21 spacings (nanoparticle concentration) and a constant heating time of $2 \mu \mathrm{s}$. The results 22 show that the $3 \mu \mathrm{m}$ box approaches the limit of the heated sphere in an infinite medium, 23 and increasing the box size does not decrease the temperature profile of the liquid. 24 Conversely, decreasing the fluid domain size to approach $L_{\text {fluid }} \approx 0.75 \mu \mathrm{m}$ resulted in 25 significant thermal boundary overlap and fluid heating. The results show that the 26 sustained heating of a large number of dispersed nanoparticles can produce a significant 27 global fluid temperature rise. 

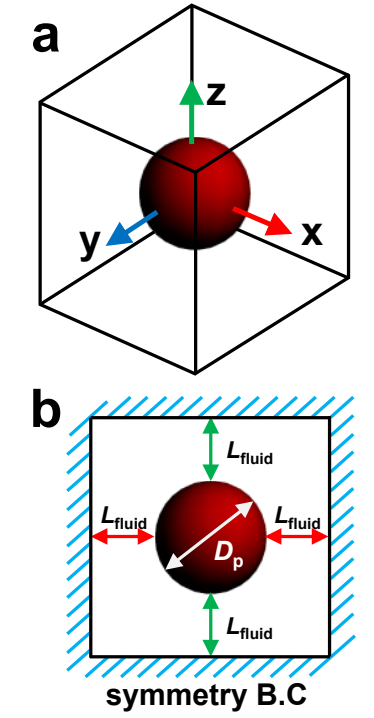

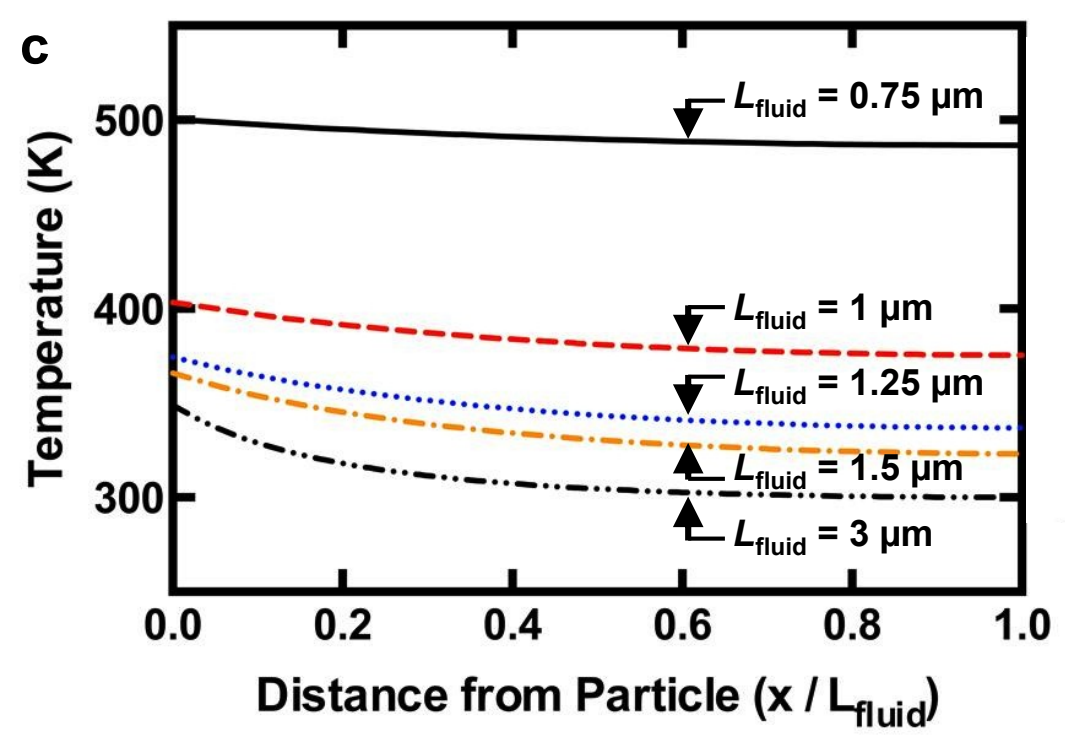

2 Figure7. (a) Isometric view of the COMSOL model domain showing the heated nanoparticle (red sphere) in a fluid box surrounding it. (b) Side view of the fluid domain showing the critical simulation dimensions: particle diameter $\left(D_{\mathrm{p}}\right)$, and distance from the particle edge to the domain boundary $\left(L_{\text {fluid }}\right)$. (c) Mean fluid temperature as a function of normalized distance from the nanoparticle wall for 5 different fluid domain sizes. As the fluid domain decreased in size, the fluid temperature increased due to the larger thermal boundary overlapping between particles.

A natural question is to compare using nanofluids versus surface absorbers for generating vapor. Our work has focused on evaporating water at temperatures below $100^{\circ} \mathrm{C}$, and is expected to outperform (5-10\%) a surface absorber designed for similar applications (see Supporting Information). Another related application is in solar boiling of water. Here, the nanofluid operates similarly to a surface absorber, since a tuned nanofluid will absorb sunlight at the surface for maximum heat concentration. A nanofluid-based absorber may have comparative advantages in high flux applications, due to its ability to increase critical heat flux[40,72]

17 In the future, it would be interesting to investigate methods to further increase the temperature of the generated vaporviavapor flow restriction. By confining the vapor escape from the nanofluid receiver, the evaporation heat transfer and overall heat transfer coefficient of the entire device decreases, increasing the temperature of the fluid within. In addition, the capability of directly generating steam at elevated pressures needs further investigation. Typically, in a power generation cycle, high temperature pressurized steam is required for efficient operation, with steam-based Rankine cycles using steam at temperatures in the range of $300-500^{\circ} \mathrm{C}$. Currently, a more suitable power application for our small-scale device is the organic Rankine cycle, which requires working fluid temperatures of only $100-200^{\circ} \mathrm{C} .[73,74]$ Another potential area for future work is developing approaches for superheating the generated steam using solar energy to high temperatures for power generation applications. In applications requiring turbines, 
1 condensing liquid from the working vapor causes erosion on the turbine blades, and

2 increases costs.

3 Another area for future work is in integrating nanofluids into current cycle designs, such 4 as a solar absorption cooling cycle. Depending on whether a system is closed-loop or 5 open loop, the fluid influx can contain respectively nanoparticles or pure fresh water. In 6 the closed-loop case such as an absorption refrigeration cycle, the nanoparticles are small 7 enough to pass through pumps, and the various concentrations of fluids can be remixed.

8 In an open-loop cycle, when operating at steady state, fresh water is required to feed the 9 receiver and balance the water vapor leaving the system. This ensures a constant 10 nanoparticle concentration. Possibly a mixing element will be needed to evenly disperse 11 the nanoparticle, but pumps in a closed-cycle can accomplish this task. In general, 12 agitation tends to decrease the aggregation of the system, as evidenced by the nanofluid 13 preparation (ultrasonication bath for dispersing). This actually increases the effectiveness 14 of the volumetric receiver, as shown in the Figure 3.

\section{Conclusion}

In summary, we demonstrated a high efficiency (69\%) nanofluids-based solar receiver for direct vapor generation, using low concentration sunlight (10 suns). At such low solar concentrations, a nanofluid solar receiver may be used in lower cost systems that do not require the use of active sun-tracking devices, although monthly repositioning may be required. Three water-based nanofluids, graphitized carbon black, graphene, and carbon black, were tested in the receiver. We experimentally demonstrated and theoretically verified that in transient situations, such as in solar vaporgeneration, the graphitized carbon black and graphenenanofluids outperformed the carbon black nanofluid by $7 \%$, after 1.5 hours of illumination. To show global fluid temperature rise as the more accurate vapor generation mechanism for nanofluids at the studies solar concentrations (1 $<C<10$ suns), we constructed heat transfer models for the receiver at the deviceand nanoparticle scales. The device scale lumped capacitance model closely predicted the bulk temperature response of the nanoparticle receiver. The particle model showed that at feasible solar concentrations and illumination times, it is highly unlikely to achieve local temperature gradients leading to nanobubble generation around the nanoparticle, as proposed previously. This work demonstrates a solar vapor generation platform that promises to be low cost and scalable for a wide-range of solar-based applications such as power generation, distillation, and sterilization.

\section{Acknowledgements}

35 We thank K. McEnaney and D. Kraemer with help operating the solar simulator, and T.

36 McClure and the Center for Materials Science and Engineering for the use of the UV-Vis

37 Spectrophotometer. This work has implications in solar steam generation, and was

38 partially supported by the Cooperative Agreement between the Masdar Institute of 39 Science and Technology, UAE and the Massachusetts Institute of Technology (MIT), 40 USA (for the steam generation). We gratefully acknowledge funding support from the 41 MIT S3TEC Center, an Energy Frontier Research Center funded by the Department of 42 Energy, Office of Science, Basic Energy Sciences under Award \# DE-FG02-09ER46577 43 (for the experimental facility). Some of the existing infrastructure used in the research 
1 was purchased under the S3TEC grant. This work was performed in part at the Center 2 for Nanoscale Systems (CNS), a member of the National Nanotechnology Infrastructure 3 Network (NNIN), which is supported by the National Science Foundation under NSF 4 award no. ECS-0335765. CNS is part of Harvard University.

\section{References}

15 [1] M.S. Bohn, K.Y. Wang, J. Sol. Energy Eng. 110 (1988) 45.

[2] B. Norton, (2014) 123.

[3] M. Thirugnanasambandam, S. Iniyan, R. Goic, Renewable Sustainable Energy Rev. 14 (2010) 312.

[4] G.T. Machinda, S.P. Chowdhury, S. Chowdhury, S. Kibaara, R. Arscott, in:, IEEE India Conference, IEEE, 1981, pp. 1-6.

[5] Y. Tian, C.Y. Zhao, Appl. Energy 104 (2013) 538.

[6] O. Neumann, A.S. Urban, J. Day, S. Lal, P. Nordlander, N.J. Halas, ACS Nano 7 (2013) 42.

23 [7] W.F. Bogaerts, C.M. Lampert, J. Mater. Sci. 18 (1983) 2847.

[8] Q.-C. Zhang, Sol. Energy Mater. Sol. Cells 62 (2000) 63.

24 [9] D. Mills, Sol. Energy 76 (2004) 19.

[10] C.E. Kennedy, 1617 (2002) 1. 
[14] H. Price, E. Lüpfert, D. Kearney, E. Zarza, G. Cohen, R. Gee, R. Mahoney, J. Sol. Energy Eng. 124 (2002) 109.

[15] A. Veeraragavan, A. Lenert, B. Yilbas, S. Al-Dini, E.N. Wang, Heat Mass Transfer 55 (2012) 556.

[16] A. Lenert, Y.S.P. Zuniga, E.N. Wang, in:, 14th International Heat Transfer Conference, Washington DC, 2010, pp. 499-508.

[17] A. Lenert, E.N. Wang, Sol. Energy 86 (2012) 253.

[18] R.A. Taylor, P. Phelan, R. Adrian, A. Gunawan, T. Otanicar, in:, ASME 2011 5th International Conference on Energy Sustainability, American Society of Mechanical Engineers, Washington DC, 2011, pp. 1927-1936.

[19] A.O. Govorov, H.H. Richardson, Nano Today 2 (2007) 30.

[20] M.T. Carlson, A.J. Green, H.H. Richardson, Nano Lett. 12 (2012) 1534.

[21] A. Sanchot, G. Baffou, R. Marty, A. Arbouet, R. Quidant, C. Girard, E. Dujardin, ACS Nano 6 (2012) 3434.

[22] H. Tyagi, P. Phelan, R. Prasher, J. Sol. Energy Eng. 131 (2009) 041004.

[23] R.A. Taylor, P.E. Phelan, T.P. Otanicar, C.A. Walker, M. Nguyen, S. Trimble, R. Prasher, J. Renewable Sustainable Energy 3 (2011) 023104.

[24] N. Arai, Y. Itaya, M. Hasatani, Sol. Energy 32 (1984) 49.

[25] T. Fend, B. Hoffschmidt, R. Pitz-Paal, O. Reutter, P. Rietbrock, Energy 29 (2004) 823.

[26] N.E. Wijeysundera, V. Thevendran, Sol. Energy 40 (1988) 127.

[27] T. Fend, R. Pitz-Paal, O. Reutter, J. Bauer, B. Hoffschmidt, Sol. Energy Mater. Sol. Cells 84 (2004) 291.

[28] R. Pitz-Paal, B. Hoffschmidt, M. Böhmer, M. Becker, Sol. Energy 60 (1997) 135.

[29] F.L. Lansing, V. Clarke, R. Reynolds, Energy 4 (1979) 685.

[30] A.O. Govorov, W. Zhang, T. Skeini, H. Richardson, J. Lee, N.A. Kotov, Nanoscale Res. Lett. 1 (2006) 84.

[31] R. Bertocchi, J. Karni, A. Kribus, Energy 29 (2004) 687.

[32] D. Han, Z. Meng, D. Wu, C. Zhang, H. Zhu, Nanoscale Res. Lett. 6 (2012) 1.

[33] F. Miller, R. Koenigsdorff, Sol. Energy Mater. 24 (1991) 210.

[34] T.P. Otanicar, P.E. Phelan, R.S. Prasher, G. Rosengarten, R.A. Taylor, J. Renewable Sustainable Energy 2 (2010) 033102.

[35] S.V. Boriskina, H. Ghasemi, G. Chen, Mater. Today 16 (2013) 375.

[36] N.J. Hogan, A.S. Urban, C. Ayala-Orozco, A. Pimpinelli, Nano Lett. (2014).

[37] Z. Fang, Y.-R. Zhen, O. Neumann, A. Polman, F.J. García de Abajo, P. Nordlander, N.J. Halas, Nano Lett. 13 (2013) 1736.

[38] E. Lukianova-Hleb, Y. Hu, L. Latterini, L. Tarpani, S. Lee, R.A. Drezek, J.H. Hafner, D.O. Lapotko, ACS Nano 4 (2010) 2109.

[39] R. Saidur, T.C. Meng, Z. Said, M. Hasanuzzaman, A. Kamyar, Heat Mass Transfer 55 (2012) 5899.

[40] S.J. Kim, I.C. Bang, J. Buongiorno, L.W. Hu, International Journal of Heat and Mass Transfer 50 (2007) 4105.

[41] S.M. You, J.H. Kim, K.H. Kim, Appl. Phys. Lett. 83 (2003) 3374.

[42] J. Lombard, T. Biben, S. Merabia, Phys. Rev. Lett. 112 (2014) 105701.

[43] S. Baral, A.J. Green, M.Y. Livshits, A.O. Govorov, H.H. Richardson, ACS Nano 
[44] G. Baffou, J. Polleux, H. Rigneault, S. Monneret, J. Phys. Chem. C 118 (2014) 4890.

[45] V. Kotaidis, C. Dahmen, G. von Plessen, F. Springer, A. Plech, J. Chem. Phys. 124 (2006) 184702.

[46] P. Keblinski, D.G. Cahill, A. Bodapati, C.R. Sullivan, T.A. Taton, J. Appl. Phys. 100 (2006) 054305.

[47] M.J. Montes, A. Abánades, J.M. Martínez-Val, Sol. Energy 83 (2009) 679.

[48] B. Kelly, U. Hermann, M. Hale, in:, Proceedings of Solar Energy Forum: the Power to Choose, Washington DC, 2001, pp. 393-398.

[49] V. Quaschning, W. Ortmanns, in:, ISES Solar World Congress 2003, Goteborg, SE, 2003, pp. 14-19.

[50] G. Resch, T. Faber, R. Haas-EEG, M. Ragwitz, A. Held, I. KonstantinaviciuteLEI, Report (D4) of the IEE Project OPTRES: Assessment and Optimisation of Renewable Support Schemes in the European Electricity Market (2006) 35.

[51] J.J. O'Gallagher, Nonimaging Optics in Solar Energy, Synthesis Lectures on Energy and the Environment: Technology, Science, and Society, 2008.

[52] G.J. Kolb, C.K. Ho, T.R. Mancini, J.A. Gary, Power Tower Technology Roadmap and Cost Reduction Plan, Sandia National Lab, 2011.

[53] O. Neumann, C. Feronti, A.D. Neumann, A. Dong, K. Schell, B. Lu, E. Kim, M. Quinn, S. Thompson, N. Grady, Proc. Natl. Acad. Sci. U. S. a. 110 (2013) 11677.

[54] C.-Y. Su, A.-Y. Lu, Y. Xu, F.-R. Chen, A.N. Khlobystov, L.-J. Li, ACS Nano 5 (2011) 2332.

[55] V. Trisaksri, S. Wongwises, Renewable Sustainable Energy Rev. 11 (2007) 512.

[56] J. Buongiorno, D.C. Venerus, N. Prabhat, T. McKrell, J. Townsend, R. Christianson, Y.V. Tolmachev, P. Keblinski, L.-W. Hu, J.L. Alvarado, I.C. Bang, S.W. Bishnoi, M. Bonetti, F. Botz, A. Cecere, Y. Chang, G. Chen, H. Chen, S.J. Chung, M.K. Chyu, S.K. Das, R. Di Paola, Y. Ding, F. Dubois, G. Dzido, J. Eapen, W. Escher, D. Funfschilling, Q. Galand, J. Gao, P.E. Gharagozloo, K.E. Goodson, J.G. Gutierrez, H. Hong, M. Horton, K.S. Hwang, C.S. Iorio, S.P. Jang, A.B. Jarzebski, Y. Jiang, L. Jin, S. Kabelac, A. Kamath, M.A. Kedzierski, L.G. Kieng, C. Kim, J.-H. Kim, S. Kim, S.H. Lee, K.C. Leong, I. Manna, B. Michel, R. Ni, H.E. Patel, J. Philip, D. Poulikakos, C. Reynaud, R. Savino, P.K. Singh, P. Song, T. Sundararajan, E. Timofeeva, T. Tritcak, A.N. Turanov, S. Van Vaerenbergh, D. Wen, S. Witharana, C. Yang, W.-H. Yeh, X.-Z. Zhao, S.-Q. Zhou, J. Appl. Phys. 106 (2009) 094312.

[57] W.T. Xie, Y.J. Dai, R.Z. Wang, K. Sumathy, Renewable Sustainable Energy Rev. 15 (2011) 2588.

[58] R.A. Taylor, P.E. Phelan, T.P. Otanicar, R. Adrian, R. Prasher, Nanoscale Res. Lett. 6 (2011) 1.

[59] W. Yu, H. Xie, J. Nanomater. 2012 (2012) 1.

[60] L. Fedele, L. Colla, S. Bobbo, S. Barison, F. Agresti, Nanoscale Res. Lett. 6 (2011) 1.

[61] Y. Hwang, J.-K. Lee, J.-K. Lee, Y.-M. Jeong, S.-I. Cheong, Y.-C. Ahn, S.H. Kim, Powder Technol. 186 (2008) 145.

[62] M. Mehrali, E. Sadeghinezhad, S.T. Latibari, S.N. Kazi, M. Mehrali, M.N.B.M. 
3 [64] B.J. Stagg, T.T. Charalampopoulos, Combust. Flame 94 (1993) 381.

4 [65] T.L. Bergman, A.S. Levine, F.P. Incropera, D.P. Dewitt, Fundamentals of Heat

[66] D.M. Kerslake, The Stress of Hot Environments, Reissue, Cambridge University Press, 2011.

[67] R.-H. Chen, T.X. Phuoc, D. Martello, Heat Mass Transfer 53 (2010) 3677.

[68] S. Lee, P.E. Phelan, L. Dai, R. Prasher, A. Gunawan, R.A. Taylor, Appl. Phys. Lett. 104 (2014) 151908.

[69] S.T. Huxtable, D.G. Cahill, S. Shenogin, L. Xue, R. Ozisik, P. Barone, M. Usrey, M.S. Strano, G. Siddons, M. Shim, P. Keblinski, Nat. Mater. 2 (2003) 731.

[70] P. Keblinski, J.A. Eastman, D.G. Cahill, Mater. Today 8 (2005) 36.

[71] G. Baffou, P. Berto, E. Bermúdez Ureña, R. Quidant, S. Monneret, J. Polleux, H. Rigneault, ACS Nano 7 (2013) 6478.

16 [72] H. Kim, Nanoscale Res. Lett. 6 (2011) 415.

17 [73] S. Quoilin, M. Orosz, H. Hemond, V. Lemort, Sol. Energy 85 (2011) 955.

18 [74] A.N.M. Delgado-Torres, L. García-Rodríguez, Energy Convers. Manage. 51 (2010) 2846. 
1

2 TOC Graphic

3

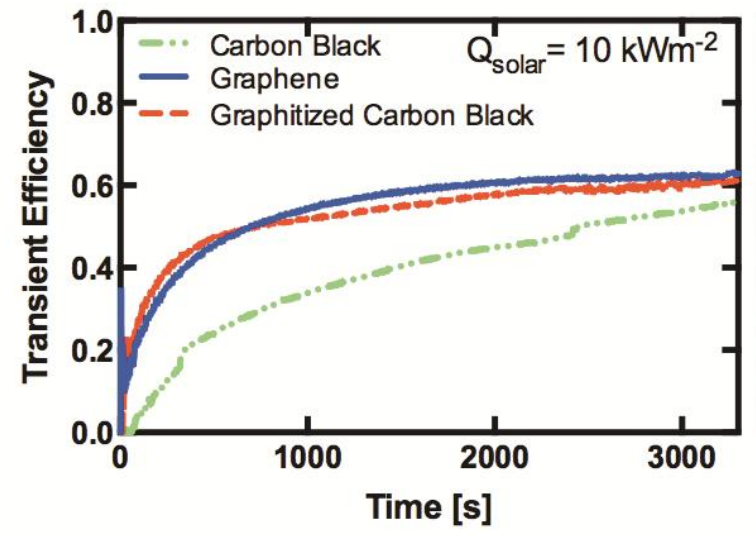

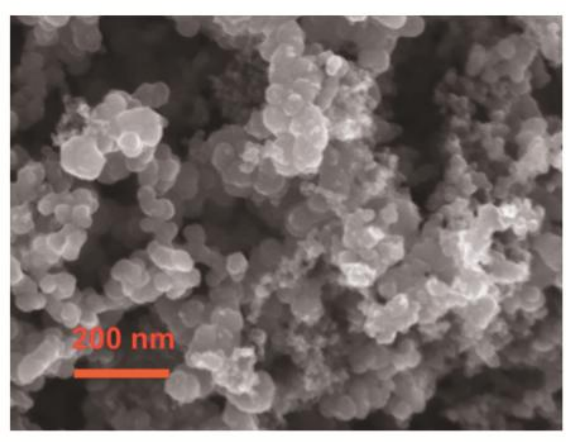

4 

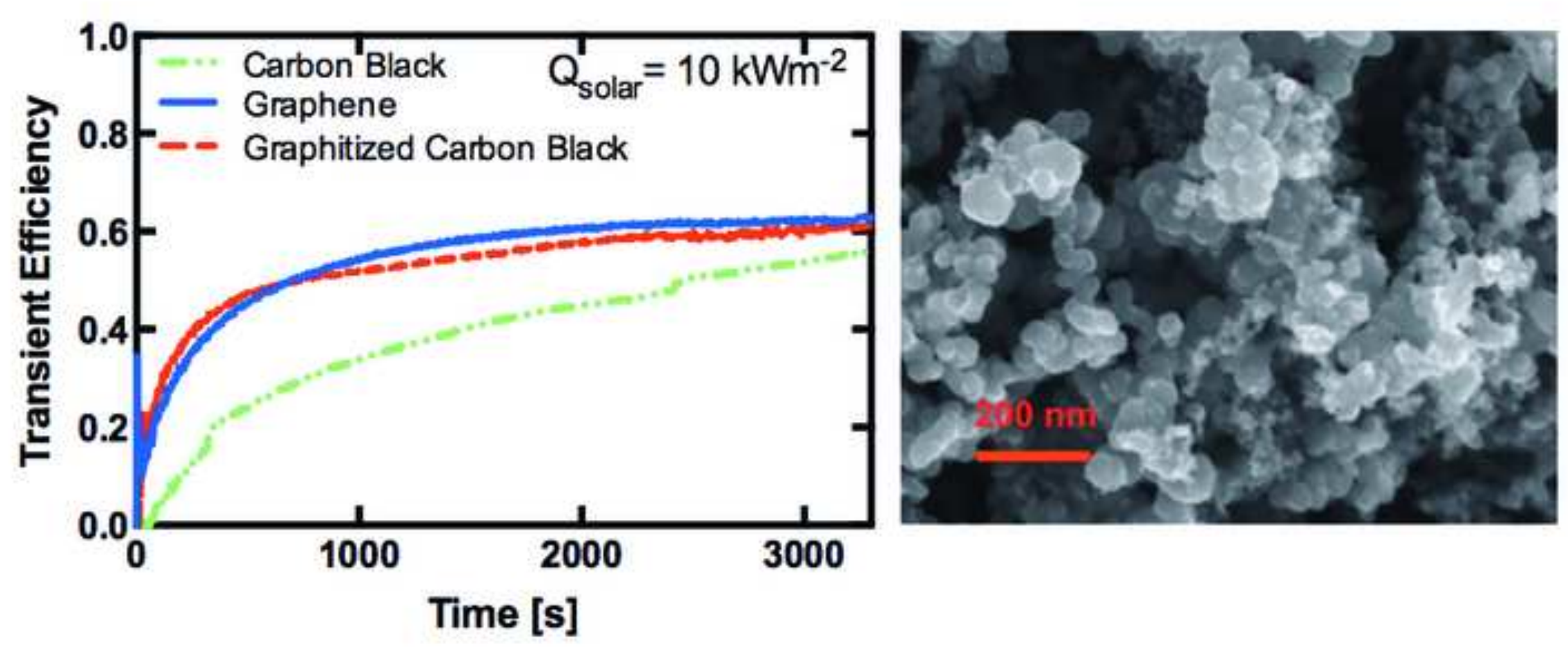